\title{
A new combinatorial interpretation of a $q$-analogue of the Lah numbers
}

\author{
Jim Lindsay, Toufik Mansour and Mark Shattuck
}

The Lah numbers $L(n, k)$ are the connection constants between the rising factorial and falling factorial polynomial bases and count partitions of $n$ distinct objects into $k$ blocks, where objects within a block are ordered (termed Laguerre configurations). In this paper, we consider the $q$-Lah numbers defined as the connection constants between the comparable bases of polynomials obtained by replacing each positive integer $n$ with $n_{q}=1+q+\cdots+q^{n-1}$ and provide a new combinatorial interpretation for these numbers by describing a statistic on Laguerre configurations for which they are the generating function. We describe some other algebraic properties of these numbers and can provide combinatorial explanations in several instances using our interpretation. A further generalization involving a second parameter may also be given.

AMS 2000 SUBJECT CLASSifiCATiOns: Primary 05A15; secondary 05A05. KEYWORDS AND PHRASES: Laguerre configuration, Lah numbers, $q$-analogue, statistic.

\section{Introduction}

We'll use the following notational conventions: $\mathbb{N}:=\{0,1,2, \ldots\}, \mathbb{P}:=\{1$, $2, \ldots\},[0]:=\varnothing$, and $[n]:=\{1, \ldots, n\}$ for $n \in \mathbb{P}$. Empty sums take the value 0 and empty products the value 1 , with $0^{0}:=1$. The letter $q$ denotes an indeterminate, with $0_{q}:=0, n_{q}:=1+q+\cdots+q^{n-1}$ for $n \in \mathbb{P}, 0_{q} !:=1$, $n_{q} !:=1_{q} 2_{q} \cdots n_{q}$ for $n \in \mathbb{P}$, and $\left(\begin{array}{l}n \\ k\end{array}\right)_{q}:=\frac{n_{q} !}{k_{q} !(n-k)_{q} !}$ for $n \in \mathbb{N}$ and $0 \leqslant k \leqslant n$. The binomial coefficient $\left(\begin{array}{l}n \\ k\end{array}\right)$ is equal to zero if $k$ is a negative integer or if $0 \leqslant n<k$.

Let $\mathcal{L}(n, k)$ denote the set of all distributions of $n$ balls, labelled $1, \ldots, n$, among $k$ unlabelled, contents-ordered boxes, with no box left empty. Garsia and Remmel [2] call such distributions Laguerre configurations. See also [8] and [12]. If $L(n, k):=|\mathcal{L}(n, k)|$, then $L(n, 0)=\delta_{n, 0}, \forall n \in \mathbb{N}, L(n, k)=0$ if 
$0 \leqslant n<k$, and

$$
L(n, k)=\frac{n !}{k !}\left(\begin{array}{l}
n-1 \\
k-1
\end{array}\right), \quad 1 \leqslant k \leqslant n,
$$

or, equivalently,

$$
L(n, k)=L(n-1, k-1)+(n-1+k) L(n-1, k), \quad \forall n, k \in \mathbb{P},
$$

with the initial conditions $L(n, 0)=\delta_{n, 0}$ and $L(0, k)=\delta_{0, k} \forall n, k \in \mathbb{N}$. The numbers $L(n, k)$ are called Lah numbers, after Ivo Lah [5], who introduced them as the connection constants in the polynomial identities

(3) $x(x+1) \cdots(x+n-1)=\sum_{k=0}^{n} L(n, k) x(x-1) \cdots(x-k+1), \quad \forall n \in \mathbb{N}$.

For applications to physics, see, e.g., [9] and [10] and the references therein, and see Section 3.3 of [6] for a related sequence of linear differential equations.

In this paper, we consider the $q$-Lah numbers $L_{q}(n, k)$ that are the connection constants in the polynomial identities

$$
\begin{aligned}
x & \left(x+1_{q}\right) \cdots\left(x+(n-1)_{q}\right) \\
& =\sum_{k=0}^{n} L_{q}(n, k) x\left(x-1_{q}\right) \cdots\left(x-(k-1)_{q}\right), \quad \forall n \in \mathbb{N},
\end{aligned}
$$

a simple $q$-generalization of (3). See, e.g., Garsia and Remmel [2] and Wagner [14] for other examples of $q$-Lah numbers. Miceli and Remmel [7] have given a general rook theory model which supplies combinatorial interpretations to much more general product formulas than those of the form of (4). Here, we provide a new combinatorial interpretation of the polynomial $L_{q}(n, k)$ given by (4) above by defining a statistic on $\mathcal{L}(n, k)$ for which it is the generating function. In addition, we describe several interesting properties of $L_{q}(n, k)$, including an expression which reduces to (1) when $q=1$, the evaluations at $q=0$ and $q=-1$, and the evaluation of the derivative at $q=1$. A further generalization involving a second parameter may also be given in terms of a joint distribution polynomial for two statistics on $\mathcal{L}(n, k)$. We observe that the $p, q$-analogue of the rook theory model of Miceli and Remmel [7] also applies to our $p, q$-analogue. 


\section{A statistic on Laguerre configurations}

Let $\mathcal{L}(n, k)$ denote the set of all distributions of $n$ balls, labelled $1,2, \ldots, n$, among $k$ unlabelled, contents-ordered boxes, with no box left empty, and let $L(n, k):=|\mathcal{L}(n, k)|$. In this section, we define a statistic on $\mathcal{L}(n, k)$ for which $L_{q}(n, k)$ given by (4) above is the generating function.

Let $\delta \in \mathcal{L}(n, k)$. We now describe a representation for $\delta$ which we will use below. Suppose first that we express the ordered contents of each box of $\delta$ by a word in $[n]$, and then arrange these words in a sequence $W_{1}, \ldots, W_{k}$ in increasing order of their least elements. For each $i$, note that the word $W_{i}$ is a permutation, which we express using standard cycle form, i.e., cycles are ordered by increasing smallest elements and each cycle is written with its smallest element in the first position. Once this is done, write out the words $W_{i}$ from left to right, inserting a bar between consecutive words. We will call this the standard form of $\delta$. As an illustration, if $\delta \in \mathcal{L}(12,5)$ is given by

$$
\lfloor 3,9,4\lfloor 8,1,10\lfloor 2\lfloor\leq, 7,5,12\lfloor\mid 11
$$

then its standard form would be

$$
\delta=(1,8)(10)|(2)|(3)(4,9)|(5,6,7)(12)|(11) .
$$

We define a statistic on $\mathcal{L}(n, k)$ as follows. Suppose $\delta \in \mathcal{L}(n, k)$ is written in standard form.

Definition 2.1. An ordered pair $(i, j)$ is an element inversion if $i>j$ and $i$ occurs to the left of $j$ in $\delta$, where $i$ is not the first (= smallest) element of any cycle of $\delta$.

Definition 2.2. An ordered pair $(i, j)$ is a block inversion if $i>j$ and $i$ occurs to the left of $j$ in $\delta$, where $i$ is the smallest element of some cycle of $\delta$ and $j$ is the smallest element of some block.

Let $i n v_{e}(\delta)$ and $i n v_{b}(\delta)$ denote the number of element and the number of block inversions of $\delta$, respectively, and let $i n v^{*}(\delta)=i n v_{e}(\delta)+i n v_{b}(\delta)$. For example, if $\delta$ is given by (5) above, then $i n v_{e}(\delta)=6+3=9$, inv $v_{b}(\delta)=$ $3+1=4$, and $i n v^{*}(\delta)=9+4=13$. When $k=1$, the $i n v^{*}$ statistic on $\mathcal{L}(n, k)$ is equivalent to a statistic first defined by Carlitz [1] on the symmetric group $\mathcal{S}_{n}$ and later studied [11].

Given $n$ and $k, 0 \leqslant k \leqslant n$, let $a_{q}(n, k)$ denote the distribution polynomial for the $i n v^{*}$ statistic on $\mathcal{L}(n, k)$, i.e.,

$$
a_{q}(n, k):=\sum_{\delta \in \mathcal{L}(n, k)} q^{i n v^{*}(\delta)} .
$$


The $a_{q}(n, k)$ satisfy a simple recurrence which generalizes (2) above.

Theorem 2.1. If $n, k \in \mathbb{P}$ and $1 \leqslant k \leqslant n$, then

$$
a_{q}(n, k)=a_{q}(n-1, k-1)+\left[(n-1)_{q}+k_{q}\right] a_{q}(n-1, k),
$$

with $a_{q}(0, k)=\delta_{k, 0}$ and $a_{q}(n, 0)=\delta_{n, 0}$ for all $n, k \in \mathbb{N}$.

Proof. The boundary values are clear, so assume $n, k \in \mathbb{P}$. The first term on the right-hand side of (7) gives the total $i n v^{*}$-weight of all the members of $\mathcal{L}(n, k)$ in which the element $n$ occupies a block by itself. The total weight of all members of $\mathcal{L}(n, k)$ in which $n$ occurs by itself in a cycle but occupies a block with at least one member of $[n-1]$ is $\left(1+q+\cdots+q^{k-1}\right) a_{q}(n-1, k)=$ $k_{q} a_{q}(n-1, k)$, since placing the cycle $(n)$ in the $i^{t h}$ block from the left within a member of $\mathcal{L}(n-1, k)$ creates $k-i$ additional block inversions. Finally, the total weight of all the members of $\mathcal{L}(n, k)$ in which $n$ occurs in a cycle with at least one member of $[n-1]$ is $\left(1+q+\cdots+q^{n-2}\right) a_{q}(n-1, k)$, since $n$ contributes $n-i-1$ additional element inversions whenever it is placed directly after the $i^{t h}$ number from the left within a member of $\mathcal{L}(n-1, k)$.

Let $L_{q}(n, k), 0 \leqslant k \leqslant n$, be defined as the connection constants between the $q$-rising factorial basis $\left(\prod_{i=0}^{n}\left(x+i_{q}\right)\right)_{n \geqslant 0}$ and the $q$-falling factorial basis $\left(\prod_{i=0}^{n}\left(x-i_{q}\right)\right)_{n \geqslant 0}$, as in (4) above. From (3) and (4), it is clear that $L_{q}(n, k)$ reduces to $L(n, k)$ when $q=1$.

Theorem 2.2. If $n, k \in \mathbb{N}$ and $0 \leqslant k \leqslant n$, then

$$
L_{q}(n, k)=a_{q}(n, k) .
$$

Proof. From (4), the equality is obvious for $n=0, k=0$, or $n=1$. To complete the proof, we need to show that the $L_{q}(n, k)$ satisfy recurrence (7) when $n \geqslant 2$. From (4), we may write

$$
\begin{aligned}
& \sum_{k=1}^{n} L_{q}(n, k) x\left(x-1_{q}\right) \cdots\left(x-(k-1)_{q}\right)=x\left(x+1_{q}\right) \cdots\left(x+(n-1)_{q}\right) \\
& \quad=\left(x+(n-1)_{q}\right) \sum_{k=0}^{n-1} L_{q}(n-1, k) x\left(x-1_{q}\right) \cdots\left(x-(k-1)_{q}\right) \\
& \quad=\sum_{k=0}^{n-1} L_{q}(n-1, k) x\left(x-1_{q}\right) \cdots\left(x-(k-1)_{q}\right)\left[\left(x-k_{q}\right)+\left((n-1)_{q}+k_{q}\right)\right]
\end{aligned}
$$




$$
\begin{aligned}
= & \sum_{k=0}^{n-1} L_{q}(n-1, k) x\left(x-1_{q}\right) \cdots\left(x-k_{q}\right) \\
& +\sum_{k=0}^{n-1}\left[(n-1)_{q}+k_{q}\right] L_{q}(n-1, k) x\left(x-1_{q}\right) \cdots\left(x-(k-1)_{q}\right) \\
= & \sum_{k=1}^{n} L_{q}(n-1, k-1) x\left(x-1_{q}\right) \cdots\left(x-(k-1)_{q}\right) \\
& +\sum_{k=1}^{n}\left[(n-1)_{q}+k_{q}\right] L_{q}(n-1, k) x\left(x-1_{q}\right) \cdots\left(x-(k-1)_{q}\right) .
\end{aligned}
$$

Comparing coefficients yields the requested result.

We may give a couple of additional recurrences satisfied by the $L_{q}(n, k)$.

Proposition 2.3. If $n, k \in \mathbb{P}$ and $1 \leqslant k \leqslant n$, then

$$
L_{q}(n, k)=\sum_{j=0}^{k}\left[(n-1-j)_{q}+(k-j)_{q}\right] L_{q}(n-1-j, k-j) .
$$

Proof. Fix $j, 0 \leqslant j \leqslant k$, and consider all the members of $\mathcal{L}(n, k)$, expressed in standard form, for which $n-j$ is the largest element occupying a block with at least one other element of $[n]$. Then elements $n-j+1, n-j+2, \ldots$, $n$ must all go in their own blocks, and regarding the placement of $n-j$, there are $n-1-j$ possible positions within cycles directly following an element of $[n-1-j]$ and $k-j$ possible positions for the cycle $(n-j)$. Thus, the total $i n v^{*}$-weight of all such members of $\mathcal{L}(n, k)$ is

$$
\left[(n-1-j)_{q}+(k-j)_{q}\right] L_{q}(n-1-j, k-j),
$$

and summing over all $j$ yields recurrence (9).

Proposition 2.4. If $n, k \in \mathbb{P}$ and $1 \leqslant k \leqslant n$, then

$$
L_{q}(n, k)=\sum_{j=k}^{n} L_{q}(j-1, k-1) \prod_{i=j}^{n-1}\left(i_{q}+k_{q}\right) .
$$

Proof. Note that the total $i n v^{*}$-weight of all the members of $\mathcal{L}(n, k)$ for which $j$ is the smallest element of the $k^{t h}$ block is $L_{q}(j-1, k-1) \prod_{i=j}^{n-1}\left(i_{q}+\right.$ $k_{q}$ ). For $j$ would start the first cycle of the $k^{\text {th }}$ block (and thus contribute 
nothing towards $i n v^{*}$, while regarding the placement of each $i \in\{j+1$, $j+2, \ldots, n\}$, there are $i-1$ possible positions within cycles following elements of $[i-1]$ and $k$ possible positions for the cycle $(i)$. Summing over all $j$ yields recurrence $(10)$.

\subsection{The case $q=0$}

Setting $q=0$ in $L_{q}(n, k)$ gives the following result.

Proposition 2.5. If $n, k \in \mathbb{P}$, then there are $2^{n-k}\left(\begin{array}{l}n-1 \\ k-1\end{array}\right)$ members of $\mathcal{L}(n, k)$ which contain neither an element inversion nor a block inversion.

Proof. Note that a distribution $\delta \in \mathcal{L}(n, k)$, expressed in standard form, has no element or block inversions if and only if the members of $[n]$ appear in the natural order in a left-to-right scan of $\delta$. There are then $\left(\begin{array}{l}n-1 \\ k-1\end{array}\right)$ ways to choose the blocks (upon placing $k-1$ dividers in $n-1$ slots) and, once this is done, $2^{n-k}$ ways to choose the cycles within the blocks.

Let $L_{q}(n):=\sum_{k=0}^{n} L_{q}(n, k)$. If $q=1$, then $L_{q}(n)$ reduces to $L(n):=$ $|\mathcal{L}(n)|$, where $\mathcal{L}(n)$ denotes the set of all Laguerre configurations of size $n$. If $q=0$, then $L_{q}(n)$ reduces to $3^{n-1}$ if $n \geqslant 1$, by Proposition 2.5. Alternatively, note that the numbers $1,2, \ldots, n$ must appear in order from left to right within a member of $\mathcal{L}(n)$ containing no element or block inversions; thus, concerning each of the $n-1$ positions separating $1,2, \ldots, n$, one may either insert a block divider, insert a cycle divider, or leave empty.

Let $L(n, k ; r)=\left.\frac{1}{r !} \frac{d^{r}}{d q^{r}} L_{q}(n, k)\right|_{q=0}$. Theorems 2.1 and 2.2 imply

$$
L(n, k ; r)=L(n-1, k-1 ; r)+\sum_{i=0}^{r}\left(\delta_{k>i}+\delta_{n>i+1}\right) L(n-1, k ; r-i)
$$

with $L(n, n ; r)=\delta_{r=0}$, where $\delta_{S}=1$ if the statement $S$ holds and $\delta_{S}=0$ otherwise.

Theorem 2.6. We have

(i) $L(n, k ; 0)=2^{n-k}\left(\begin{array}{c}n-1 \\ k-1\end{array}\right)$ for all $1 \leqslant k \leqslant n$,

(ii) $L(n, k ; 1)=\frac{2 n k-3 k-n+1}{k} 2^{n-k-1}\left(\begin{array}{l}n-2 \\ k-1\end{array}\right)$ for all $1 \leqslant k \leqslant n-1$,

(iii) $L(n, k ; 2)=\frac{(k+1)\left(4 k^{2}-7 k+2\right)-\left(2 k^{3}+2 k^{2}+1\right) n+\left(2 k^{2}-1\right) n^{2}}{k(k+1)} 2^{n-k-2}\left(\begin{array}{l}n-2 \\ k-1\end{array}\right)$ for all $2 \leqslant k \leqslant n-1$, with $L(n, 1 ; 2)=(n-3)(n+2) 2^{n-4}$ for all $n \geqslant 3$. 
Proof. (i) We give another proof of Proposition 2.5 above. Recurrence (11) for $r=0$ gives $L(n, k ; 0)=L(n-1, k-1 ; 0)+2 L(n-1, k ; 0)$ if $n \geqslant 2$ and $k \geqslant 1$, with $L(1,1 ; 0)=1$. This recurrence may be written as

$$
2^{-(n-k)} L(n, k ; 0)=2^{-(n-k)} L(n-1, k-1 ; 0)+2^{-(n-k-1)} L(n-1, k ; 0),
$$

which, by induction, implies $2^{-(n-k)} L(n, k ; 0)=\left(\begin{array}{c}n-1 \\ k-1\end{array}\right)$. Thus, $L(n, k ; 0)=$ $2^{n-k}\left(\begin{array}{l}n-1 \\ k-1\end{array}\right)$, as claimed.

(ii) When $r=1$, recurrence (11) implies for $n \geqslant 3$,

$$
L(n, k ; 1)=L(n-1, k-1 ; 1)+2 L(n-1, k ; 1)+\left(1+\delta_{k>1}\right) L(n-1, k ; 0),
$$

with $L(2, k ; 1)=0$ for $k=1,2$. If $k=1$, this is

$$
L(n, 1 ; 1)=2 L(n-1,1 ; 1)+L(n-1, k ; 0),
$$

which, by (i), implies $L(n, 1 ; 1)=2^{n-2}\left(\begin{array}{c}n-2 \\ 1\end{array}\right)$. For $k \geqslant 2$, we have

$$
L(n, k ; 1)=L(n-1, k-1 ; 1)+2 L(n-1, k ; 1)+2 L(n-1, k ; 0) .
$$

Multiplying the last equation by $y^{k}$, summing over all $k=2,3, \ldots, n$, and using (i) implies

$$
\begin{aligned}
& L_{n}^{1}(y)-2^{n-2}(n-2) y \\
& \quad=(2+y) L_{n-1}^{1}(y)-2^{n-2}(n-3) y+2 \sum_{k=2}^{n-1} 2^{n-k-1}\left(\begin{array}{c}
n-2 \\
k-1
\end{array}\right) y^{k},
\end{aligned}
$$

for all $n \geqslant 3$ with $L_{2}^{1}(y)=0$, where $L_{n}^{1}(y):=\sum_{k=1}^{n} L(n, k ; 1) y^{k}$. Solving this recurrence relation (for example, by Maple) yields

$$
L_{n}^{1}(y)=2^{n-1}-2((n-2) y-1)(2+y)^{n-2},
$$

which implies $L(n, k ; 1)=\frac{2 n k-3 k-n+1}{k} 2^{n-k-1}\left(\begin{array}{c}n-2 \\ k-1\end{array}\right)$ for all $n \geqslant 2$ and $k=$ $1,2, \ldots, n-1$.

(iii) Arguments similar to those used in the case $r=1$ apply to the case $r=2$.

Comparable, though more complicated, expressions may be given for $L(n, k ; r)$ in the cases when $r=3$ and $r=4$. Combinatorially, statement (ii) 
of Theorem 2.6 asserts that the members of $\mathcal{L}(n, k)$ having a single inversion (either element or block) number

$$
2^{n-k-1}\left((2 n-3)\left(\begin{array}{c}
n-2 \\
k-1
\end{array}\right)-\left(\begin{array}{c}
n-1 \\
k
\end{array}\right)\right), \quad 1 \leqslant k \leqslant n-1
$$

Summing this over $k$ implies that the members of $\mathcal{L}(n)$ having a single inversion number

$$
2^{n-1}+2(n-3) 3^{n-2}, \quad n \geqslant 2 .
$$

We can provide combinatorial explanations for the last two formulas.

\section{Combinatorial proofs}

Proof. We prove only the first formula, the proof of the second being similar. Suppose $n \geqslant 3$. Let $\mathcal{L}(n, k ; j) \subseteq \mathcal{L}(n, k)$ comprise those members with $j$ inversions altogether (counting both element and block). We first count those members of $\mathcal{L}(n, k ; 1)$ having an element inversion. Let $3 \leqslant i \leqslant n$ and suppose an element inversion within a member of $\mathcal{L}(n, k ; 1)$ is caused by the number $i$ (necessarily coming to the left of $i-1$ in standard form). Note that we may produce such a configuration by first forming a member of $\mathcal{L}(n-1, k ; 0)$ (using the elements $[n]-\{i\}$ ), writing it in standard form, and then adding $i$ directly before $i-1$ (when scanned left-to-right) in such a way so that $i$ is not first in a cycle. (That is, add $i$ directly before $i-1$ within its cycle if $i-1$ does not start a cycle and add $i$ to the end of the first cycle to the left of $i-1$ if it does.) Thus, by (i) in Theorem 2.6, there are $2^{n-k-1}(n-2)\left(\begin{array}{l}n-2 \\ k-1\end{array}\right)$ members of $\mathcal{L}(n, k ; 1)$ having an element inversion.

To complete the proof of the first formula above, we must show that the members of $\mathcal{L}(n, k ; 1)$ having a block inversion number

$$
2^{n-k-1}\left((n-2)\left(\begin{array}{l}
n-2 \\
k-1
\end{array}\right)-\left(\begin{array}{c}
n-2 \\
k
\end{array}\right)\right), \quad k \geqslant 2 .
$$

To form a member of $\mathcal{L}(n, k ; 1)$ with a block inversion in which the element $i$, $3 \leqslant i \leqslant n$, is responsible, take a member $\lambda \in \mathcal{L}(n-1, k ; 0)$ (on the set $[n]-\{i\})$, write it in standard form, and then add the 1-cycle $(i)$ to the block directly preceding the block containing the element $i-1$. This cannot 
be done in the case when $3 \leqslant i \leqslant n-k+1$ and $i-1$ occurs in the first block of $\lambda$. Note that for each $i$, the total number of such $\lambda$ is

$$
\sum_{j=i-1}^{n-k} 2^{j-1} \cdot\left(\begin{array}{c}
n-j-2 \\
k-2
\end{array}\right) 2^{n-j-k}=2^{n-k-1}\left(\begin{array}{c}
n-i \\
k-1
\end{array}\right),
$$

by (i), where $j$ denotes the number of elements of $[n]-\{i\}$ in the first block of $\lambda$. Subtracting these configurations, we see that the members of $\mathcal{L}(n, k ; 1)$ having a block inversion total

$$
\begin{aligned}
& \sum_{i=3}^{n} 2^{n-k-1}\left(\begin{array}{l}
n-2 \\
k-1
\end{array}\right)-\sum_{i=3}^{n-k+1} 2^{n-k-1}\left(\begin{array}{c}
n-i \\
k-1
\end{array}\right) \\
& =2^{n-k-1}(n-2)\left(\begin{array}{c}
n-2 \\
k-1
\end{array}\right)-2^{n-k-1}\left(\begin{array}{c}
n-2 \\
k
\end{array}\right),
\end{aligned}
$$

by (i), as requested.

Summing (iii) in Theorem 2.6 above over $k, 1 \leqslant k \leqslant n-1$, implies that the members of $\mathcal{L}(n)$ having exactly two inversions altogether of either kind number

$$
(2 n-1) 2^{n-2}+\left(2 n^{2}-8 n-4\right) 3^{n-3}, \quad n \geqslant 3 .
$$

One may provide a combinatorial proof of this formula along similar lines to those above.

\subsection{The case $q=-1$}

Using recurrence (7) at $q=-1$, and noting $m_{q}=[m$ is odd] if $m \in \mathbb{N}$ when $q=-1$, one may obtain Table 1 for the $L_{-1}(n, k)$ values when $0 \leqslant k \leqslant n \leqslant$ 8. The $L_{-1}(n, k)$ occur as sequence A173410 in [13]. From Table 1, certain values of $L_{-1}(n, k)$ are easily deduced, though there does not seem to be a simple closed form for all $n$ and $k$. For example, we have $L_{-1}(n, 1)=$ $L_{-1}(n, 2)=2^{\left\lfloor\frac{n}{2}\right\rfloor}$ if $n \geqslant 2$ and $L_{-1}(n, n-1)=2\left\lfloor\frac{n}{2}\right\rfloor$ if $n \geqslant 1$. If $k=3$, we have $L_{-1}(2 n, 3)=(3 n-4) 2^{n-1}$ for $n \geqslant 2$ and $L_{-1}(2 n+1,3)=(3 n-2) 2^{n-1}$ for $n \geqslant 1$. Let $s=\left\lfloor\frac{k-1}{2}\right\rfloor$ and $t=\left\lfloor\frac{n}{2}\right\rfloor-\left\lfloor\frac{k-1}{2}\right\rfloor$, where $k>0$ is fixed and $n>k$. Using recurrence (7) when $q=-1$, one may show in general that $L_{-1}(n, k)$ is of the form a polynomial in $n$ of degree $s$ times the factor $2^{t}$.

We can find an explicit formula for the generating function of the $L_{-1}(n, k)$. 
Table 1: The numbers $L_{-1}(n, k)$ for $0 \leqslant k \leqslant n \leqslant 8$

\begin{tabular}{rrrrrrrrrr}
\hline & $k=0$ & 1 & 2 & 3 & 4 & 5 & 6 & 7 & 8 \\
\hline$n=0$ & 1 & & & & & & & & \\
1 & 0 & 1 & & & & & & & \\
2 & 0 & 2 & 1 & & & & & & \\
3 & 0 & 2 & 2 & 1 & & & & & \\
4 & 0 & 4 & 4 & 4 & 1 & & & & \\
5 & 0 & 4 & 4 & 8 & 4 & 1 & & & \\
6 & 0 & 8 & 8 & 20 & 12 & 6 & 1 & & \\
7 & 0 & 8 & 8 & 28 & 20 & 18 & 6 & 1 & \\
8 & 0 & 16 & 16 & 64 & 48 & 56 & 24 & 8 & 1 \\
\hline
\end{tabular}

Theorem 2.7. The generating function

$$
A(x, y)=1+\sum_{n \geq 1} \sum_{k=1}^{n} L_{-1}(n, k) x^{n} y^{k}
$$

is given by

$$
A(x, y)=\frac{1-2 x^{2}-x^{2} y^{2}+x y+2 x^{3} y^{2}+2 x^{2} y-x^{3} y^{3}}{1-2 x^{2}-2 x^{2} y^{2}-2 x^{4} y^{2}+x^{4} y^{4}} .
$$

Proof. Denote the $a_{q}(n, k)$ defined by (6) above by $a_{n, k}$. Let $A_{n}(y)$ be the generating function for the $a_{n, k}$, that is, $A_{n}(y)=\sum_{k=0}^{n} a_{n, k} y^{k}$. Rewriting the recurrence of $a_{n, k}$ in the statement of Theorem 2.1 yields for $n \geqslant 1$,

$$
A_{n}(y)=y A_{n-1}(y)+\frac{1-q^{n-1}}{1-q} A_{n-1}(y)+\frac{A_{n-1}(y)-A_{n-1}(q y)}{1-q},
$$

with the initial condition $A_{0}(y)=1$. Now define $A(x, y)=1+\sum_{n \geq 1} A_{n}(y) x^{n}$. Rewriting the above recurrence relation in terms of generating function $A(x, y)$ implies

$$
\begin{aligned}
A(x, y)= & 1+x y A(x, y)+\frac{x}{1-q}(A(x, y)-A(q x, y)) \\
& +\frac{x}{1-q}(A(x, y)-A(x, q y)) .
\end{aligned}
$$

Replacing $(x, y)$ by $(-x, y),(x,-y)$ and $(-x,-y)$, and substituting $q=-1$ into (13), yields the following system of equations

$$
(1-x y-x) A(x, y)=1-\frac{x}{2}(A(x,-y)+A(-x, y))
$$




$$
\begin{aligned}
(1+x y+x) A(-x, y) & =1+\frac{x}{2}(A(-x,-y)+A(x, y)), \\
(1+x y-x) A(x,-y) & =1-\frac{x}{2}(A(x, y)+A(-x,-y)), \\
(1-x y+x) A(-x,-y) & =1+\frac{x}{2}(A(-x, y)+A(x,-y)),
\end{aligned}
$$

which implies

$$
A(x, y)=\frac{1-2 x^{2}-x^{2} y^{2}+x y+2 x^{3} y^{2}+2 x^{2} y-x^{3} y^{3}}{1-2 x^{2}-2 x^{2} y^{2}-2 x^{4} y^{2}+x^{4} y^{4}}
$$

as claimed.

Taking $y=1$ in (12) gives

$$
A(x, 1)=\frac{1+x-x^{2}+x^{3}}{1-4 x^{2}-x^{4}}=\sum_{n \geqslant 0} f_{\left\lfloor\frac{3 n}{2}\right\rfloor} x^{n},
$$

which implies the following explicit formula for the row sums $L_{-1}(n):=$ $\sum_{k=0}^{n} L_{-1}(n, k)$.

Corollary 2.8. If $n \in \mathbb{N}$, then

$$
L_{-1}(n)=f_{r},
$$

where $r=\left\lfloor\frac{3 n}{2}\right\rfloor$ and $f_{n}$ denotes the Fibonacci sequence defined by $f_{n}=$ $f_{n-1}+f_{n-2}$ if $n \geqslant 2$, with $f_{0}=f_{1}=1$.

We can provide a combinatorial explanation of formula (14).

\section{Combinatorial proof of Corollary 2.8}

Proof. First note that Corollary 2.8 asserts that the members of $\mathcal{L}(n)$ having even $i n v^{*}$ value outnumber those having odd $i n v^{*}$ value by $f_{r}$ for all $n$. Thus one may establish (14) by first identifying a subset $\mathcal{L}^{*}(n)$ of $\mathcal{L}(n)$ having cardinality $f_{r}$, all of whose members have even $i n v^{*}$ value, along with an inv $v^{*}$-parity changing involution of $\mathcal{L}(n)-\mathcal{L}^{*}(n)$.

Let $\mathcal{L}^{*}(n) \subseteq \mathcal{L}(n)$ consist of those configurations formed in the inductive manner as follows, $n \geqslant 2$ :

(i) If $\lambda \in \mathcal{L}^{*}(n-2)$ comprises an odd number of blocks, then add the elements $n-1, n$ as $\{(n-1, n)\},\{(n-1),(n)\}$, or $\{(n-1)\},\{(n)\}$, or as either $(n-1, n)$ or $(n-1),(n)$ to the current rightmost block. 
(ii) If $\lambda \in \mathcal{L}^{*}(n-2)$ comprises an even number of blocks, then add the elements $n-1, n$ as $\{(n-1, n)\},\{(n-1),(n)\}$, or $\{(n-1)\},\{(n)\}$.

(iii) $\mathcal{L}^{*}(0)$ is the singleton set comprising the empty configuration and $\mathcal{L}^{*}(1)=\{(1)\}$.

Then all members of $\mathcal{L}^{*}(n)$ clearly have even $i n v^{*}$-parity. It may be verified, upon considering even and odd cases, that $\left|\mathcal{L}^{*}(n)\right|=f_{r}$ by showing that if $a_{n}:=\left|\mathcal{L}^{*}(n)\right|$, then $a_{n}$ satisfies the recurrence $a_{n}=4 a_{n-2}+a_{n-4}, n \geqslant 4$, along with the initial conditions $a_{0}=a_{1}=1, a_{2}=3$ and $a_{3}=5$.

Given $\delta \in \mathcal{L}^{*}(n)$ in standard form and $i \in[n]$, let $\delta_{[i]}$ denote the member of $\mathcal{L}(i)$ (in standard form) obtained by writing the elements of $[i]$ in the order as they appear within the cycles and blocks of $\delta$ (for example, if $\delta$ is given by (5) above and $i=6$, then $\left.\delta_{[6]}=(1)|(2)|(3)(4) \mid(5,6)\right)$. To construct an $i n v^{*}$-parity changing involution $g$, let $\lambda \in \mathcal{L}(n)-\mathcal{L}^{*}(n)$ and $i_{0}$, $0 \leqslant i_{0} \leqslant\left\lfloor\frac{n}{2}\right\rfloor-1$, be the smallest number $i$ such that the elements $n-2 i$ and $n-2 i-1$ are not added to $\lambda_{[n-2 i-2]}$ in the manner described above for membership in $\mathcal{L}^{*}(n)$. The involution $g$ requires several cases to specify it completely. For simplicity, we take $i_{0}=0$ and assume that $\lambda_{[n-2]}$ has an even number of blocks (the other cases being similar). If neither $n-1$ nor $n$ occurs as a 1-cycle in $\lambda$, or exactly one belongs to a block by itself, then let $g(\lambda)$ be the distribution obtained by switching $n-1$ and $n$ within $\lambda$, leaving the other members of $[n]$ undisturbed. Next, suppose that at least one of $\{n-1, n\}$ occurs as a 1-cycle in $\lambda$, with neither occurring in a block by itself. In this case, if, say $(n)$, belongs to block $2 \ell-1$ (from the left) for some $\ell$, then move this cycle to block $2 \ell$, and if it belongs to block $2 \ell$, then move it to block $2 \ell-1$ (move $(n)$, if it occurs, otherwise move $(n-1)$ ).

Note that the above mapping does not change the $i n v^{*}$-parity in the case where $n$ occurs in block $2 \ell$ for some $\ell$ not as a 1-cycle and $n-1$ occurs in either block $2 \ell-1$ or $2 \ell$ as a 1 -cycle or in the case where the 2 -cycle $(n-1, n)$ occurs in either block $2 \ell-1$ or $2 \ell$. If the number of elements of $[n-2]$ occurring in block $2 \ell$ is even, then cyclicly shifting $n$ through the positions directly after these elements produces an equal number of distributions having even and odd $i n v^{*}$ values. Furthermore, the case where the cycle $(n-1, n)$ occurs in block $2 \ell$ pairs off with the case where it occurs in block $2 \ell-1$. If the number of elements of $[n-2]$ occurring in block $2 \ell$ is odd, then cyclicly shifting $n$ through the positions directly after these elements as well as after $n-1$ produces an equal number of distributions having even and odd $i n v^{*}$-parity. 


\subsection{The derivative at $q=1$}

In this section, we'll find an explicit formula for the derivative in $q$ of $L_{q}(n, k)$ evaluated at $q=1$, which gives the sum of the $i n v^{*}$ values taken over all of the members of $\mathcal{L}(n, k)$. We will need the following lemma.

Lemma 2.9. If $k \in \mathbb{P}$, then

$$
\sum_{j=0}^{k}(-1)^{j}\left(\begin{array}{l}
k \\
j
\end{array}\right)_{q} q^{\left(\begin{array}{c}
j+2 \\
2
\end{array}\right)-(j+1)(k+1)}=0
$$

Proof. Induct on $k$, the $k=1$ case clear. To complete the induction, substitute the identity,

$$
\left(\begin{array}{l}
k \\
j
\end{array}\right)_{q}=\left(\begin{array}{c}
k-1 \\
j-1
\end{array}\right)_{q}+q^{j}\left(\begin{array}{c}
k-1 \\
j
\end{array}\right)_{q}
$$

into the sum on the left-hand side of $(15)$ for each $j, 1 \leqslant j \leqslant k$.

By convention, we'll take $L_{q}(n, k)=0$ if $n, k \in \mathbb{P}$, with $k>n$. Note that the recurrence $(7)$ continues to hold for all $n, k \in \mathbb{P}$. We now give an explicit formula for the $L_{q}(n, k)$.

Theorem 2.10. If $n, k \in \mathbb{P}$, then

$$
L_{q}(n, k)=\sum_{j=1}^{k}\left(\frac{j_{q}\left(j_{q}+1_{q}\right) \cdots\left(j_{q}+(n-1)_{q}\right)}{\prod_{i=0, i \neq j}^{k}\left(j_{q}-i_{q}\right)}\right) .
$$

Proof. We induct on $n$ and first prove (16) when $n=1$. If $k=1$, both sides are 1 , so to complete the $n=1$ case, we must show

$$
\sum_{j=1}^{k} \frac{1}{\prod_{i=1, i \neq j}^{k}\left(j_{q}-i_{q}\right)}=0, \quad \forall k \geqslant 2 .
$$

Observe next that $a_{q}-b_{q}=q^{b}(a-b)_{q}$, if $a>b \geqslant 1$, which implies

$$
\prod_{i=1, i \neq j}^{k}\left(j_{q}-i_{q}\right)=(-1)^{k-j} q^{j(k-j)+\left(\begin{array}{l}
j \\
2
\end{array}\right)(k-j)_{q} !(j-1)_{q} !}
$$


If $k \geqslant 2$, we then have

$$
\begin{aligned}
\sum_{j=1}^{k} \frac{1}{\prod_{i=1, i \neq j}^{k}\left(j_{q}-i_{q}\right)} & =\sum_{j=1}^{k} \frac{(-1)^{k-j} q^{j(j-k)-\left(\begin{array}{c}
j \\
2
\end{array}\right)}}{(k-j)_{q} !(j-1)_{q} !} \\
& =\frac{(-1)^{k-1}}{(k-1)_{q} !} \sum_{j=1}^{k}(-1)^{j-1}\left(\begin{array}{c}
k-1 \\
j-1
\end{array}\right)_{q} q^{\left(\begin{array}{c}
j+1 \\
2
\end{array}\right)-j k} \\
& =0
\end{aligned}
$$

by Lemma 2.9, which establishes (17) and thus the $n=1$ case. The induction is now easily completed upon assuming $n \geqslant 2$ and $k \geqslant 1$ and using the recurrence $L_{q}(n, k)=L_{q}(n-1, k-1)+\left[(n-1)_{q}+k_{q}\right] L_{q}(n-1, k)$.

Letting $q=1$ on the right-hand side of (16) gives

$$
\sum_{j=1}^{k} \frac{\prod_{i=0}^{n-1}(j+i)}{\prod_{i=0, i \neq j}^{k}(j-i)}=\frac{n !}{k !} \sum_{j=1}^{k}(-1)^{k-j}\left(\begin{array}{c}
j+n-1 \\
j-1
\end{array}\right)\left(\begin{array}{l}
k \\
j
\end{array}\right)=\frac{n !}{k !}\left(\begin{array}{l}
n-1 \\
k-1
\end{array}\right)
$$

by identity (5.25) on p. 169 of [4], whence (16) generalizes (1).

Taking the derivative of both sides of (16) with respect to $q$, letting $q=1$, and noting

$$
\left.\frac{d}{d q} j_{q}\right|_{q=1}=\left(\begin{array}{l}
j \\
2
\end{array}\right)
$$

yields

$$
\left.\frac{d}{d q} L_{q}(n, k)\right|_{q=1}=\sum_{j=1}^{k} \frac{\prod_{i=0}^{n-1}(j+i)}{\prod_{i=0, i \neq j}^{k}(j-i)}\left(\sum_{i=0}^{n-1} \frac{\left(\begin{array}{l}
j \\
2
\end{array}\right)+\left(\begin{array}{l}
i \\
2
\end{array}\right)}{j+i}-\sum_{i=0, i \neq j}^{k} \frac{\left(\begin{array}{l}
j \\
2
\end{array}\right)-\left(\begin{array}{l}
i \\
2
\end{array}\right)}{j-i}\right),
$$

which implies the following result.

Theorem 2.11. If $n, k \in \mathbb{P}$, then the total inv ${ }^{*}$ value of all the members of $\mathcal{L}(n, k)$ is given by

$$
\frac{n !}{k !} \sum_{j=1}^{k}(-1)^{k-j}\left(\begin{array}{c}
j+n-1 \\
j-1
\end{array}\right)\left(\begin{array}{l}
k \\
j
\end{array}\right)\left(\sum_{i=0}^{n-1} \frac{\left(\begin{array}{l}
j \\
2
\end{array}\right)+\left(\begin{array}{l}
i \\
2
\end{array}\right)}{j+i}-\sum_{i=0, i \neq j}^{k} \frac{\left(\begin{array}{l}
j \\
2
\end{array}\right)-\left(\begin{array}{l}
i \\
2
\end{array}\right)}{j-i}\right) .
$$

Since

$$
\sum_{i=0, i \neq j}^{k} \frac{\left(\begin{array}{l}
j \\
2
\end{array}\right)-\left(\begin{array}{l}
i \\
2
\end{array}\right)}{j-i}=\sum_{i=0, i \neq j}^{k} \frac{j+i-1}{2}=\frac{1}{2}\left(\left(\begin{array}{l}
k \\
2
\end{array}\right)+(k-1) j\right),
$$


the second part of the formula above may be reduced further using identity (5.25) on p. 169 of [4], though we do not see a way to simplify the first part.

\section{A further generalization}

By defining a second statistic on $\mathcal{L}(n, k)$, one may generalize the $L_{q}(n, k)$ as follows. Suppose $\delta \in \mathcal{L}(n, k)$ is written in standard form.

Definition 3.1. An ordered pair $(i, j)$ is an element permanence if $i>j$ and $i$ occurs to the right of $j$ in $\delta$, where $i$ is not the first element of any cycle of $\delta$ and $j>1$.

Definition 3.2. An ordered pair $(i, j)$ is a block permanence if $i>j$ and $i$ occurs to the right of $j$ in $\delta$ in a different block, where $i$ is the smallest element of some cycle of $\delta$, but not the smallest element of any block, and $j$ is the smallest element of some block.

Let $\operatorname{perm}_{e}(\delta)$ and $\operatorname{perm}_{b}(\delta)$ denote the number of element and the number of block permanences of $\delta$, respectively, and let $\operatorname{perm}^{*}(\delta)=\operatorname{perm}_{e}(\delta)+$ $\operatorname{perm}_{b}(\delta)$. For example, if $\delta$ is given by (5) above, then $\operatorname{perm}_{e}(\delta)=4+4+5=$ $13, \operatorname{perm}_{b}(\delta)=2+3=5$, and $\operatorname{perm}^{*}(\delta)=13+5=18$.

Given $n$ and $k, 0 \leqslant k \leqslant n$, consider the $p, q$-Lah number $L_{p, q}(n, k)$ defined by

$$
L_{p, q}(n, k):=\sum_{\delta \in \mathcal{L}(n, k)} p^{p e r m^{*}(\delta)} q^{i n v^{*}(\delta)}
$$

If $p$ and $q$ are indeterminates, then $n_{p, q}:=p^{n-1}+p^{n-2} q+\cdots+p q^{n-2}+q^{n-1}$ if $n \in \mathbb{P}$, with $0_{p, q}:=0$. Reasoning as in the prior section shows that the $L_{p, q}(n, k)$ satisfy the boundary values $L_{p, q}(n, 0)=\delta_{n, 0}$ and $L_{p, q}(0, k)=\delta_{k, 0}$, for all $n, k \in \mathbb{N}$, along with the recurrence

$$
L_{p, q}(n, k)=L_{p, q}(n-1, k-1)+\left[(n-1)_{p, q}+k_{p, q}\right] L_{p, q}(n-1, k),
$$

for all $n, k \in \mathbb{P}$, which generalizes (7). Taking $q=1$ in (19) implies that the inv $^{*}$ and perm $^{*}$ statistics are identically distributed on $\mathcal{L}(n, k)$. Using (19), one may show that the $L_{p, q}(n, k)$ are the connection constants in the polynomial identities

$$
\begin{aligned}
x\left(x+1_{p, q}\right) \cdots\left(x+(n-1)_{p, q}\right) \\
=\sum_{k=0}^{n} L_{p, q}(n, k) x\left(x-1_{p, q}\right) \cdots\left(x-(k-1)_{p, q}\right),
\end{aligned}
$$


for all $n \in \mathbb{N}$, which generalizes (4).

We conclude with an explicit formula for $L_{p, q}(n, k)$ involving determinants. Expanding the product in (20), we obtain

$$
\begin{aligned}
& \sum_{k=d}^{n} L_{p, q}(n, k) e_{k-d}\left(-1_{p, q}, \ldots,-(k-1)_{p, q}\right) \\
& \quad=e_{n-d}\left(1_{p, q}, \ldots,(n-1)_{p, q}\right)
\end{aligned}
$$

for all $n \in \mathbb{P}$ and $d=1,2, \ldots, n$, where $e_{j}\left(x_{1}, \ldots, x_{n}\right)$ is the $j^{\text {th }}$ symmetric function on the variables $x_{1}, \ldots, x_{n}$ (by convention, $e_{0}\left(x_{1}, \ldots, x_{n}\right)=1$ ). Substituting $d=1,2, \ldots, n$ into the above equation and rewriting the $n$ resulting equations in matrix form yields, by Cramer's rule, the following formula for $n \in \mathbb{P}$ and $j=0,1, \ldots, n-1$ :

$$
L_{p, q}(n, n-j)=\operatorname{det}\left(\begin{array}{lllll}
g_{n-1,0} & 0 & \cdots & 0 & h_{0} \\
g_{n-1,1} & g_{n-2,0} & \cdots & 0 & h_{1} \\
\vdots & \vdots & \ddots & \vdots & \vdots \\
g_{n-1, j} & g_{n-2, j-1} & \cdots & g_{n-j, 1} & h_{j}
\end{array}\right)
$$

where $h_{i}=e_{i}\left(1_{p, q}, \ldots,(n-1)_{p, q}\right)$ and $g_{i, j}=e_{j}\left(-1_{p, q}, \ldots,-i_{p, q}\right)$.

\section{Some final remarks}

In this section, we compare and contrast our $q$-analogue of $L(n, k)$ with other $q$-analogues which have appeared in the literature. Recall that our $L_{q}(n, k)$ is defined by the formula

$$
\begin{aligned}
x & \left(x+1_{q}\right) \cdots\left(x+(n-1)_{q}\right) \\
& =\sum_{k=1}^{n} L_{q}(n, k) x\left(x-1_{q}\right) \cdots\left(x-(k-1)_{q}\right), \quad \forall n \in \mathbb{P},
\end{aligned}
$$

and satisfies the recurrence

$$
L_{q}(n, k)=L_{q}(n-1, k-1)+\left((n-1)_{q}+k_{q}\right) L_{q}(n-1, k), \quad \forall n, k \in \mathbb{P} .
$$

It is the generating function for a statistic on $\mathcal{L}(n, k)$ which we denote by $i n v^{*}$.

We first consider the $q$-analogue $\bar{L}_{q}(n, k)$ of Garsia and Remmel [2] which can be defined as follows. Suppose that we are given a placement $P$ of $n$ 
labelled balls, with labels from $1, \ldots, n$, in $k$ unlabelled tubes. Define the word associated with each tube by reading the balls in the tubes from bottom to top. Then associate a word with the placement $P$ by ordering the words associated with the tubes by decreasing smallest elements and placing a 0 between each of the words. See Figure 1 below. Given such a word $w$, let $\operatorname{inv}(w)$ denote the number of inversions of $w$ and let $\bar{L}_{q}(n, k)$ denote the sum of $q^{i n v(w)}$ taken over all the words that arise from a placement of $n$ labelled balls in $k$ unlabelled tubes. Note that $\operatorname{inv}(w)=23+41=64$, while $\operatorname{inv}^{*}(w)=9+4=13$ for the placement illustrated below.

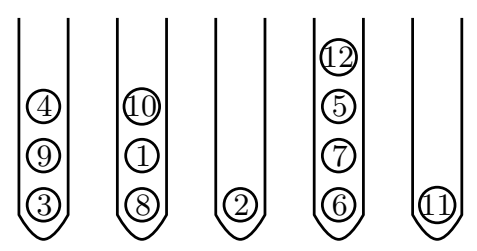

Garsia-Remmel word

Our word

$1106751203940208110 \quad 1810|2| 349|56712| 11$

Figure 1: Configuration of labelled balls in tubes.

Let $[x]_{q}:=\frac{1-q^{x}}{1-q}$ and $[x]_{q} \uparrow_{n}:=[x]_{q}[x+1]_{q} \cdots[x+n-1]_{q}$ and $[x]_{q} \downarrow_{n}:=$ $[x]_{q}[x-1]_{q} \cdots[x-n+1]_{q}$ if $n \in \mathbb{P}$, with $[x]_{q} \uparrow_{0}=[x]_{q} \downarrow_{0}:=1$. Then Garsia and Remmel [2] showed the following $q$-analogues of equations (1), (2), and (3). There is the explicit formula for $\bar{L}_{q}(n, k)$ given by

$$
\bar{L}_{q}(n, k)=q^{k(k-1)} \frac{n_{q} !}{k_{q} !}\left(\begin{array}{l}
n-1 \\
k-1
\end{array}\right)_{q}, \quad \forall n, k \in \mathbb{P} .
$$

The $\bar{L}_{q}(n, k)$ are defined by the recursions

$$
\bar{L}_{q}(n, k)=\bar{L}_{q}(n-1, k-1)+(n+k-1)_{q} \bar{L}_{q}(n-1, k), \quad \forall n, k \in \mathbb{P},
$$

along with the initial conditions $\bar{L}_{q}(n, 0)=\delta_{n, 0}$ and $\bar{L}_{q}(0, k)=\delta_{0, k} \forall n, k \in$ $\mathbb{N}$. For all $n \in \mathbb{P}$ and $x$,

$$
[x]_{q} \uparrow_{n}=\sum_{k=1}^{n} \bar{L}_{q}(n, k)[x]_{q} \downarrow_{k} .
$$


One can show that (26) has the polynomial version

$$
\begin{aligned}
& x\left(q x+1_{q}\right) \cdots\left(q^{n-1} x+(n-1)_{q}\right) \\
& \quad=\sum_{k=1}^{n} \bar{L}_{q}(n, k) x\left(\frac{x-1_{q}}{q}\right) \cdots\left(\frac{x-(k-1)_{q}}{q^{k-1}}\right), \quad \forall n \in \mathbb{P},
\end{aligned}
$$

which is of a form similar to (22).

Garsia and Remmel [3] later gave a rook theory interpretation of their $\bar{L}_{q}(n, k)$ in terms of $q$-counting rook placements on an $n \times(n-1)$ board. Using this model, one can supply completely combinatorial proofs of (24) and (25) as well as of (26) when $x$ is a non-negative integer. In contrast, only (23) above for $L_{q}(n, k)$ appears to have a combinatorial proof by our model and no $q$-analogue for (1) simpler than (16) can apparently be found.

Furthermore, this is not the first combinatorial interpretation which has been given for $L_{q}(n, k)$. As mentioned earlier, Miceli and Remmel [7] have given a general rook theory model which supplies combinatorial interpretations to much more general product formulas than those of the form of (22). In particular, Miceli and Remmel gave combinatorial interpretations for the connection coefficient expansions of the form $\prod_{i=1}^{n}\left([x]_{q} \pm\left[a_{i}\right]\right)$ in terms of expansions of the form $\prod_{i=1}^{n}\left([x]_{q} \pm\left[b_{i}\right]\right)$ for arbitrary non-negative integer sequences $a=\left(a_{1}, a_{2}, \ldots\right)$ and $b=\left(b_{1}, b_{2}, \ldots\right)$, and replacing $[x]_{q}$ by $x$ will yield equations of the form (22). See Theorem 4.1 of [7]. Another advantage of the Miceli-Remmel model is that both (22) and (23) can be given combinatorial proofs, whereas only (23) has such a proof by our model. Perhaps our interpretation for $L_{q}(n, k)$ is better suited for explaining some of the special values it assumes, as in Sections 2.1-2.3 above. A question which arises would be if our model could be used to explain (22) when, say, $x=i$ or $x=i_{q}$ for some $i \in \mathbb{N}$. In addition, the Miceli-Remmel model can be extended to a second parameter, and the $p, q$-version of their model applies to our two-variable version (see Theorem 5.1 of [7]).

Finally, the $q$-Lah numbers $\tilde{L}_{q}(n, k)$ introduced by Wagner [14] arise in the study of modular binomial lattices and are given by

$$
\tilde{L}_{q}(n, k)=\frac{n !}{k !}\left(\begin{array}{l}
n-1 \\
k-1
\end{array}\right)_{q}, \quad \forall n, k \in \mathbb{P},
$$

which generalizes (1). They may also be given as distribution polynomials for a statistic on $\mathcal{L}(n, k)$ defined as follows. Suppose $\delta=B_{1}, B_{2}, \ldots, B_{k} \in$ $\mathcal{L}(n, k)$, where the blocks of $\delta$ are arranged by increasing smallest elements. Define $\tilde{w}(\delta)=\sum_{i=1}^{k}(i-1)\left(\left|B_{i}\right|-1\right.$ ) (note $\tilde{w}$ is an analogue of a now well 
known statistic first considered by Carlitz [1] on set partitions). The polynomial $\tilde{L}_{q}(n, k)$ is then the sum of $q^{\tilde{w}(\delta)}$ taken over all of the members of $\mathcal{L}(n, k)$, see Theorem 3.3 of [12]. In contrast, the $\tilde{L}_{q}(n, k)$ do not seem to function in any interesting way as connection constants between polynomial bases nor is there any interesting generalization of recurrence (2). On the other hand, the $\tilde{L}_{q}(n, k)$ do satisfy a different type of $q$-recurrence, namely

$$
\tilde{L}_{q}(n, k)=\frac{n}{k} \tilde{L}_{q}(n-1, k-1)+n q^{k-1} \tilde{L}_{q}(n-1, k), \quad \forall n, k \in \mathbb{P},
$$

which reduces to $L(n, k)=\frac{n}{k} L(n-1, k-1)+n L(n-1, k)$ when $q=1$.

\section{Acknowledgements}

We wish to thank the anonymous referee for a thorough reading of our manuscript and for several excellent suggestions which improved its presentation. We also thank him for calling references [3] and [7] to our attention.

\section{References}

[1] Carlitz, L. (1968). Combinatorial Analysis Notes, Duke University.

[2] Garsia, A. and Remmel, J. (1980). A combinatorial interpretation of $q$ derangement and $q$-Laguerre numbers. European J. Combin. 1 47-59. MR0576766

[3] Garsia, A. and Remmel, J. (1986). q-Counting rook configurations and a formula of Frobenius. J. Combin. Theory Ser. A 41 246-275. MR0834272

[4] Graham, R., Knuth, D. and Patashnik, O. (1989). Concrete Mathematics: A Foundation for Computer Science, 2nd ed. Addison-Wesley. MR1397498

[5] Lah, I. (1955). Eine neue Art von Zahlen, ihre Eigenschaften und Anwendung in der Mathematischen Statistik. Mitteilungsbl. Math. Statist. 7 203-212. MR0074435

[6] Mansour, T. and Schork, M. (2009). The solution of the recurrence relation $f_{n}(t)=a_{n}(t) f_{n-1}(t)-b_{n}(t) \frac{d}{d t} f_{n-1}(t)$. J. Difference Equ. Appl. 15(7) 679-691. MR2535986

[7] Miceli, B.K. and Remmel, J. (2008). Augmented rook boards and general product formulas. Electron. J. Combin. 15(1) \#R85. MR2426148 
[8] Motzkin, T.S. (1971). Sorting Numbers for Cylinders and Other Classification Numbers, Proc. Sympos. Pure Math. vol. 19, American Mathematical Society, 167-176. MR0332508

[9] Parthasarathy, R. (2004). q-Fermionic numbers and their roles in some physical problems. Phys. Lett. A 326 178-186. MR2065620

[10] Schork, M. (2003). Fermionic relatives of Stirling and Lah numbers. J. Phys. A: Math. Gen. 36 10391-10398. MR2024828

[11] Shattuck, M. (2005). Parity theorems for statistics on permutations and Catalan words. Integers 5 \#A07. MR2139163

[12] Shattuck, M. and Wagner, C. (2005). Parity theorems for statistics on lattice paths and Laguerre configurations. J. Integer Seq. 8 Art. 5.5.1. MR2176968

[13] Sloane, N.J. On-Line Encyclopedia of Integer Sequences, available electronically at http://www.research.att.com/ NJAS/sequences/.

[14] Wagner, C. (1996). Generalized Stirling and Lah numbers. Discrete Math. 160 199-218. MR1417571

JIM LINDSAY

MATHEMATICS Department

University of Tennessee

KNOXVILLE, TN 37996

USA

E-mail address: lindsay@math.utk.edu

Toufik Mansour

Mathematics Department

UNIVERSITY OF HAIFA

HAIFA 31905

ISRAEL

E-mail address: toufik@math.haifa.ac.il

Mark Shattuck

Mathematics Department

UnIVERsity OF TENNEsseE

KNOXVILLE, TN 37996

USA

E-mail address: shattuck@math.utk.edu

Received March 2, 2010 